\section{Ingestão Alimentar em Estudos Epidemiológicos}

\section{Food Intake in Epidemiological Studies}

\section{Resumo}

A epidemiologia da nutrição dispõe de uma gama de metodologias para avaliar o estado nutricional. Entretanto, os métodos dietéticos são os únicos capazes de detectar a deficiência nutricional em seu estágio inicial, sendo por isso bastante utilizados em estudos epidemiológicos de associação entre exposições e eventos. Atualmente, o método dietético mais indicado para estudos epidemiológicos, principalmente aqueles envolvendo doenças crônico-degenerativas, tem sido o questionário semiquantitativo de freqüência alimentar (QSFA). Este instrumento, não diferente de outros, está sujeito à variabilidade e a erros de medida. Entretanto, estes podem ser identificados e tratados utilizando-se métodos como comparação de médias dietéticas, estudos de confiabilidade, de validação relativa e de calibração. Em estudo realizado em Bambuí-MG, o consumo de nutrientes foi avaliado por três diferentes métodos: QSFA e recordatórios 24 horas (R24) com e sem réplicas de alimentos, tendo suas médias comparadas $\mathrm{e}$ calibradas. As médias encontradas pelo QSFA foram consistentemente maiores do que as dos R24. No entanto, quando essas foram ajustadas pelo consumo calórico total não apresentaram diferenças importantes, exceto para fibras; zinco; vitaminas $\mathrm{C}$; $\mathrm{B}_{6}$, e E; e colesterol. E as médias calibradas foram, em geral, menores do que as não calibradas, sendo estatisticamente significantes para ingestão de lipídeos; fibras; zinco; vitaminas $\mathrm{C}, \mathrm{E}$ e A; ácidos graxos saturados, monoinsaturados e poliinsaturados; e colesterol.

Palavras-chave: Calibração. Erros de medida. Nutrição. Questionário semiquantitativo de freqüência alimentar. Validação relativa. Ingestão alimentar.

Centro de Pesquisas René Rachou

Fundação Oswaldo Cruz

Grupo de Pesquisa em Epidemiologia

Departamento de Medicina Preventiva e Social

Faculdade de Medicina

Universidade Federal de Minas Gerais 
Abstract

There are several approaches aiming to evaluate nutritional status available for epidemiological studies. Dietary methods are some of the most recommended because of their ability to detect nutritional deficiency in its initial stage. Currently, the Semiquantitative Food Frequency Questionnaire (SFFQ) has been chosen as the best dietary method, especially when dealing with chronic degenerative diseases. This tool, like many others, is subject to intrinsic variability and measurement errors. However, both phenomena can be identified and somehow treated through different methodologies such as comparison of dietary means, reliability studies, relative validity and calibration. In a study carried out in the town of Bambuí, nutrient intake measurements were obtained using SFFQ with photos and the 24-Hour Recall (R24) questionnaire with and without foodstuff replicas. Average nutrient intake obtained by the SFFQ was significantly higher compared to R24 questionnaires for all nutrients. However, when the averages were adjusted for total caloric intake, means were similar, except for fiber, zinc, vitamins $\mathrm{C}, \mathrm{B}_{6}$ and $\mathrm{E}$, and cholesterol consumption. The calibrated averages obtained by SFFQ were generally lower than those from the non-calibrated SFFQ and significant for lipids; fiber; zinc; vitamins $\mathrm{C}, \mathrm{E}$ and $\mathrm{A}$; saturated, monounsaturated and polyunsaturated fatty acids intake.

Keywords: Calibration. Measurement of error. Nutrition. Semi-quantitative food frequency questionnaire. Relative validation. Food intake.

\section{Introdução}

O questionário semiquantitativo de freqüência alimentar (QSFA) tem sido o melhor método de avaliação dietética para estudos epidemiológicos, dada a sua capacidade de englobar períodos longos de tempo, sua confiabilidade e validade relativa. Entretanto, por ser um questionário fechado, ou seja, os alimentos são definidos previamente, sua validade pode ser comprometida por listas incompletas ou excessivas de alimentos, devendo ser validado ou calibrado para cada população. Está também sujeito a erros de informação das freqüências e porções, o que pode prejudicar sua validade e precisão, sendo recomendado o uso de fotos e/ou medidas caseiras ${ }^{1-8}$.

O presente trabalho refere-se a uma revisão da adequação do QSFA na avaliação do consumo alimentar em estudos epidemiológicos, ilustrado com informações obtidas a partir do inquérito nutricional realizado em Bambuí, Minas Gerais ${ }^{9}$. As bases de dados do MEDLINE (Literatura Científica Internacional), LILACS (Literatura LatinoAmericana e do Caribe em Ciências da Saúde) e publicações específicas da área de $\mathrm{Nu}$ trição foram utilizadas para a pesquisa bibliográfica.

\section{Dificuldades em avaliar a ingestão alimentar}

Independente do método escolhido para quantificar a ingestão alimentar, a obtenção de dados válidos e confiáveis em estudos epidemiológicos nutricionais é tarefa difícil, uma vez que não existe um método ouro para avaliação da ingestão de alimentos e nutrientes, e os métodos utilizados estão sujeitos a variações e erros de medida.

\section{Variação Entre Indivíduos}

Uma importante fonte de variação entre indivíduos é aquela proveniente das diferenças entre sexo e idade. Os homens, via de regra, apresentam consumo mais elevado do que as mulheres, e as necessidades nutricio- 
nais são diferenciadas em faixas etárias distintas. Por isto, recomenda-se que a descrição da ingestão alimentar seja sempre estratificada por sexo e idade ${ }^{1,2}$.

\section{Variabilidade Externa: Efeitos dos Dias da Semana e Sazonalidade Alimentar}

A ingestão de nutrientes também pode variar com o dia da semana, tendendo a ser maior nos finais de semana. No entanto, este efeito é observado de forma diferencial, dependendo do nutriente. Por exemplo, naqueles em que as variações entre e intraindivíduos são grandes, tais como a vitamina A, o colesterol e o sódio, tal efeito pode não ser tão evidente. Para minimizar esta variação, pode-se ajustar o nutriente pela ingestão calórica total, conhecida como densidade de nutrientes, ou incorporar aleatoriamente todos os dias da semana no estudo quando se tratar do R24, ou ainda utilizar o QSFA $^{1}$.

Já a sazonalidade alimentar, resultante da influência das estações do ano no consumo de alimentos e nutrientes, depende do grupo populacional, do nível socioeconômico e da área geográfica em estudo ${ }^{9}$. Tem importante influência na ingestão de vitaminas A, C, ferro e calorias, em países onde a oferta de alimentos energéticos é sazonal. Pode ser minimizada pelo uso do QSFA ou por inclusão aleatória de dias representativos das estações do ano quando se tratar do R24 ${ }^{1}$.

\section{Erros de Medida em Métodos Dietéticos}

Além da variabilidade proveniente do próprio método dietético, a mensuração da ingestão alimentar, mesmo em condições ótimas de estudo, pode estar sujeita a erros de medida. Conhecer e quantificar a magnitude destes é uma recomendação em estudos epidemiológicos ${ }^{10}$. Os erros são classificados em aleatórios e sistemáticos, podendo ser intra ou entre indivíduos ${ }^{4,10}$.

No erro aleatório intra-indivíduo, as médias de ingestão obtidas a partir da reaplicação do mesmo instrumento no indi- víduo (teste-reteste) alternam em torno da ingestão real, sem seguir um padrão. Ele pode ocorrer por variação diária na ingestão ou por erro de medida ${ }^{4}$. Portanto, com o uso do QSFA este erro pode ser evitado.

No erro sistemático intra-indivíduo, as médias obtidas a partir da repetição do instrumento no mesmo indivíduo estão em torno da ingestão real, mas seguem um padrão de sub ou superestimação. Isso ocorre quando a ingestão não reflete a verdadeira média por utilizar questionário estruturado, como o QSFA, no qual algum alimento importante ou grupo de alimentos são omitidos ou mal interpretados.

No erro aleatório entre indivíduos, a ingestão está ora subestimada, ora superestimada em relação à ingestão real. Ocorre quando há poucas observações do indivíduo na presença do erro aleatório intra-indivíduo ou quando o erro sistemático intraindivíduo é randomicamente distribuído.

Por fim, no erro sistemático entre indivíduos, a ingestão do grupo segue um padrão diferente do aleatório em relação à ingestão real. Resulta do erro sistemático intra-indivíduo, devido à omissão de alimentos em um questionário estruturado ou por utilizar tabelas de composição de alimentos inadequadas $^{4}$.

Como forma de minimizar ou eliminar esses erros, recomenda-se para os intra-indivíduos o uso do QSFA ou múltiplos R24 para avaliar o consumo alimentar. E, para os entre indivíduos, a validação relativa e a calibração do QSFA, metodologias estas discutidas à frente.

\section{A ingestão alimentar em estudos epidemiológicos}

Considerando a complexidade na obtenção de medidas válidas da ingestão alimentar em estudos epidemiológicos, muito se tem investido na adequação de instrumentos. Entre os mais utilizados destaca-se o QSFA, considerado atualmente o que melhor expressa o consumo alimentar, principalmente quando o objetivo é a associação entre o consumo de alimentos ou de nutri- 
entes e doenças crônicas ${ }^{1,3,4,7,9}$.

Várias metodologias têm sido propostas a fim de corrigir as possíveis variações e erros provenientes desse método, permitindo assim alcançar sua adequação ${ }^{4}$.

\section{Adequação de Questionários Semiquantitativos de Freqüência Alimentar (QSFA)}

O desempenho do QSFA (instrumento teste) tem sido avaliado através da sua comparação com um método referência, geralmente o R24 ou o registro alimentar com a pesagem de alimentos. Dentre estes, o método referência mais aplicável em estudos epidemiológicos tem sido o R24, devido à sua facilidade de aplicação em grupos populacionais variados, inclusive aqueles de pouca ou nenhuma escolaridade. A adequação inclui análises de comparações de médias dietéticas, estudos de confiabilidade, de validação relativa e de calibração $0^{1,3,4,7,8,10,11}$.

\section{Estudos de Comparação de Médias Dietéticas}

Consiste na análise comparativa das médias de nutrientes obtidas a partir do método teste e do referência. Pode ser externa ou interna. Externa, quando os dados obtidos a partir do QSFA são comparados com inquéritos nacionais. Esta modalidade ainda é pouco utilizada em nosso meio, dada a escassez de estudos nacionais regulares avaliando o consumo, embora ainda possam ser feitas comparações com inquéritos realizados em populações semelhantes ${ }^{4}$.

Já a interna refere-se à comparação das médias de nutrientes obtidas a partir do QSFA com aquelas obtidas a partir do método de referência dentro de uma mesma amostra ${ }^{4,9}$. Como exemplo, tem-se o inquérito nutricional realizado em Bambuí, um município com 15.000 habitantes, situado no centrooeste do estado de Minas Gerais. Neste estudo, em uma amostra de 98 indivíduos, a ingestão alimentar foi avaliada pelo QSFA associado a fotos e recordatórios 24 horas (R24) com e sem réplicas de alimentos. O QSFA foi aplicado face a face e, após cerca de um mês, os recordatórios. Todos os procedimentos foram realizados aplicados no domicílio dos entrevistados. As médias dos nutrientes obtidas pelo QSFA foram maiores $(\mathrm{p} \leq 0,05)$ do que aquelas apresentadas pelos R24. Entretanto, as médias entre os dois modelos de recordatórios apresentaram valores semelhantes, sendo que o R24 com réplicas apresentou médias superiores para vitaminas em geral e minerais como fósforo, ferro e zinco (Tabela 1) ${ }^{9}$. Quando ajustadas pelo consumo calórico total, as médias obtidas tanto pelo QSFA como pelos recordatórios foram similares, exceto para fibras, vitaminas $\mathrm{C}, \mathrm{B}_{6}$ e E que se apresentavam superestimadas, e para o colesterol e zinco, subestimados. O uso de réplicas permitiu maior aproximação entre as médias ajustadas obtidas pelo QSFA e pelo recordatório (Tabela 2).

\section{Estudos de Confiabilidade ou \\ Reprodutibilidade}

A confiabilidade ou reprodutibilidade refere-se à capacidade do instrumento para fornecer resultados semelhantes em diferentes repetições. Entre os fatores que podem afetar a confiabilidade destacam-se os intervalos de tempo entre as entrevistas, a variação entre os entrevistadores e a variação intra-indivíduo do entrevistado.

Quando o intervalo de tempo é pequeno, como poucos dias ou semanas, o indivíduo pode recordar as informações e repetilas na próxima entrevista, aumentando a confiabilidade. Por outro lado, quando são usados intervalos longos, pode haver mudanças no consumo alimentar, reduzindo a confiabilidade ${ }^{4}$.

Os estudos de confiabilidade do QSFA geralmente incluem coeficientes de correlação entre nutrientes. Como exemplo serão citados alguns estudos.

No trabalho de Byers et al. ${ }^{12}$ foi encontrado um baixo coeficiente de correlação entre os instrumentos para vitamina $A$ $(\mathrm{r}=0,29)$. Willett et al. ${ }^{3}$, Byers et al. ${ }^{13} \mathrm{e}$ Pietinen et al. ${ }^{4}$ obtiveram coeficientes melhores e próximos $(0,52,0,60$ e 0,53 , respectivamente), e 


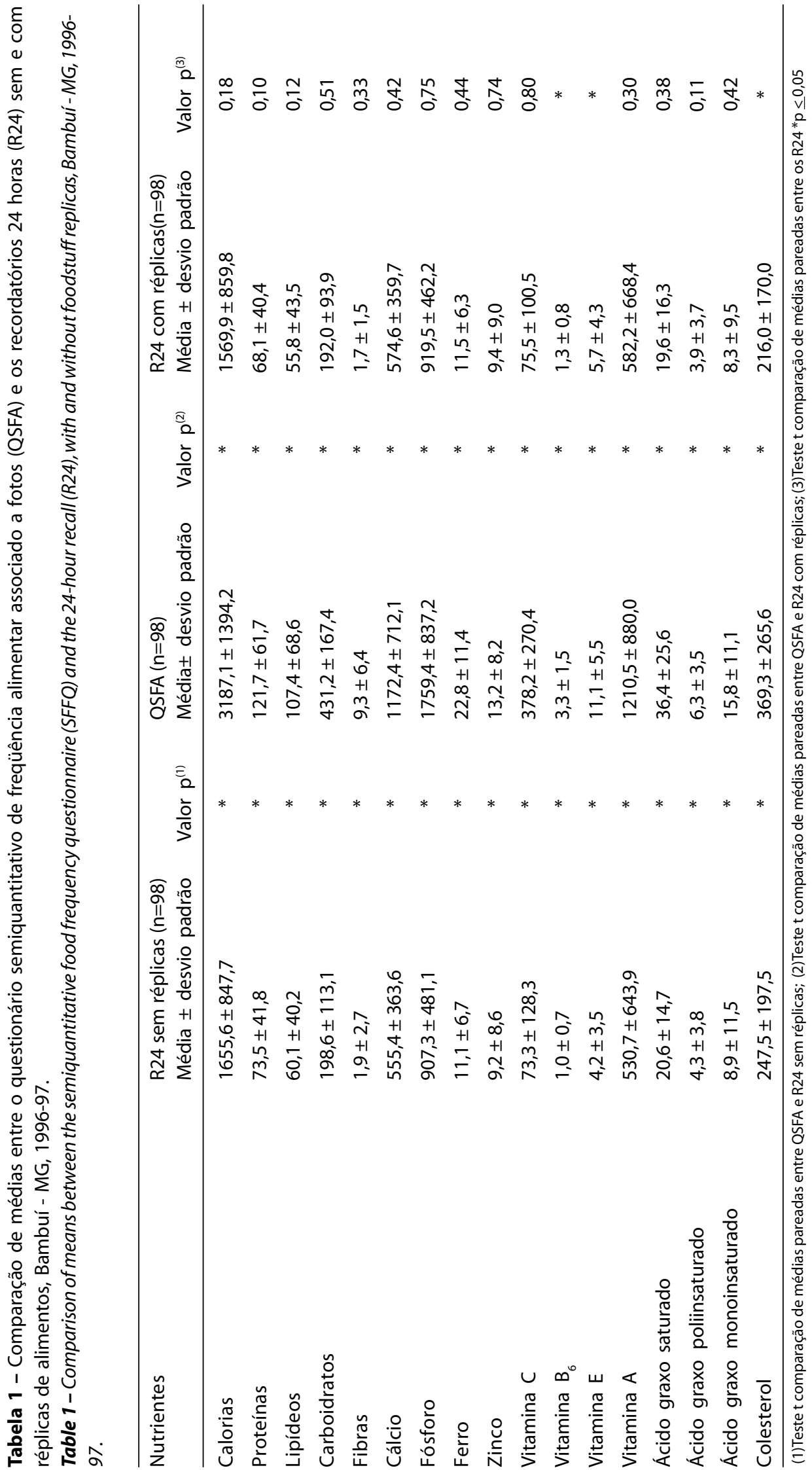




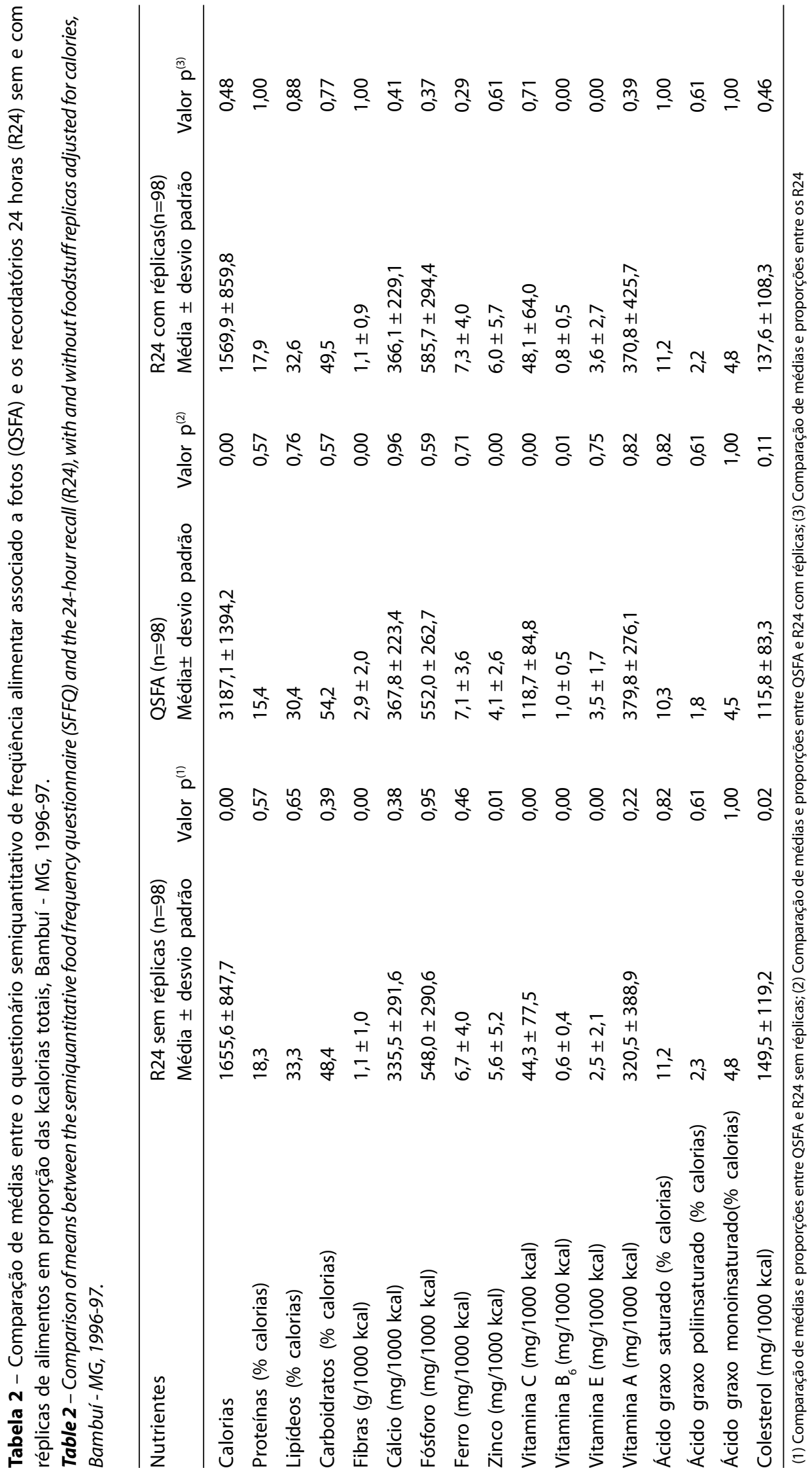


Engle et al. uma correlação de 0,75 entre os instrumentos ${ }^{15}$. É interessante ressaltar que, no estudo de Byers et al., quando foi obtido um coeficiente tão baixo $(\mathrm{r}=0,29)^{12}$, o objetivo era testar fontes de vitamina A. No entanto, o intervalo entre as repetições foi longo (17 a 25 anos), o que parece ter favorecido uma grande variação intra-indivíduo, reduzindo portanto a confiabilidade. Por outro lado, Engle et al. encontraram uma correlação elevada para a vitamina $\mathrm{A}(\mathrm{r}=0,75)$ trabalhando com um intervalo de apenas um mês ${ }^{15}$, tempo este reduzido em relação aos demais estudos. Neste caso, o intervalo poderia ter contribuído para aumentar a confiabilidade.

Considerando a proteína, foram encontradas baixas e moderadas correlações, variando de $0,25^{15}$ a $0,57^{16}$ segundo os grupos pesquisados, como idosos e diabéticos, respectivamente.

As correlações para o consumo de álcool foram elevadas, variando de 0,79 em mulheres $^{15}$ a 0,88 em ambos os sexos ${ }^{14}$, independente do intervalo de tempo entre as repetições. Este achado parece sugerir que o consumo de álcool é mais marcante e/ou constante do que o de nutrientes, provavelmente devido à sua conotação social, proporcionando maior confiabilidade ${ }^{6}$.

Para os lipídeos, os valores encontrados foram bastante próximos, sendo que Byers et al. encontraram um coeficiente de $0,50^{13} \mathrm{e}$ Hansson et al. de $0,58^{17}$. Já para os poliinsaturados, a correlação foi baixa, $0,38^{11}$, e para a colesterol foi $0,79^{16}$.

Vale ressaltar que a maioria desses estudos utilizou grupos populacionais específicos, tais como idosos ${ }^{12}$, enfermeiras ${ }^{3}$, homens $^{14}$, mulheres ${ }^{11}$ e diabéticos ${ }^{16}$. Tal variabilidade impede a comparabilidade e a extrapolação dos resultados para a população em geral.

$\mathrm{Na}$ interpretação desses estudos, uma baixa confiabilidade não significa necessariamente um instrumento não replicável, mas sugere apenas que o mesmo não forneceu medidas estáveis ao longo do tempo. Por outro lado, uma moderada ou alta confiabilidade pode ser resultante de erros, como o sistemático intra-indivíduo, e não de uma boa reprodutibilidade. Os estudos de confiabilidade, portanto, não são capazes de aferir a validade, mas sim a estabilidade da medida em diferentes repetições ${ }^{17}$. Para verificar a acurácia dos instrumentos dietéticos, são recomendados estudos de validação relativa.

\section{Estudos de Validação Relativa}

Para verificar a validade de instrumentos dietéticos usa-se a validação relativa, dada a inexistência de um padrão-ouro que forneça a real ingestão alimentar. A validação relativa é definida como o método que avalia se o instrumento mede o que realmente pretende medir, isto é, se a ingestão obtida pelo QSFA encontra-se próxima ao real. Ela é realizada pela comparação da ingestão obtida pelo método teste com aquela mais próxima possível do real, obtida pelo método referência, no caso o R24 ou o registro alimentar. Por esses instrumentos referirem-se apenas à ingestão alimentar de um único dia, devem ser reaplicados a fim de contemplar a sazonalidade alimentar e a variação dietética, garantindo assim sua aproximação com a ingestão real ${ }^{18}$.

Os estudos de validação relativa têm sido realizados desde os anos $60^{19}$, sendo interessante notar que, mesmo eles exigindo métodos estatísticos e computacionais complexos e lidando com a pouca tecnologia da época, os resultados encontrados foram semelhantes aqueles obtidos recentemente. No entanto, essas pesquisas só avançaram a partir de 1985, quando Willett et al. realizaram um estudo prospectivo para identificar fatores de risco para o câncer ${ }^{3}$, criando o tipo de QSFA mais utilizado atualmente. A necessidade do uso desse instrumento em outras populações intensificou a realização de estudos de validação relativa e calibração.

Como exemplos de estudos de validação relativa, destacam-se aqueles realizados por Browe et al. ${ }^{19}$, incluindo apenas indivíduos do sexo masculino, o de Willett et al. $^{3}$, incluindo enfermeiras, Ocké et al..$^{20} \mathrm{em}$ ambos os sexos, e o de Sichieri et al..$^{5} \mathrm{em}$ funcionários 
de uma universidade brasileira. Os métodos referência usados variaram desde história dietética, registro alimentar ao R24, estando também presentes outros marcadores como índice de massa corporal, excreção urinária de nitrogênio e dosagens bioquímicas. As correlações variaram desde valores muito baixos, como o encontrado por Sichieri et al. para a vitamina $A(r=0,15$ entre professores) ${ }^{5}$, até valores muito altos, como para o consumo de álcool em mulheres no estudo de Ocké et al. $(\mathrm{r}=0,87)^{20}$.

A proteína, o nutriente mais descrito, foi encontrada subdividida em vegetal e total. Correlação moderada, com coeficiente de $0,66^{19}$, foi encontrada para a proteína vegetal, e coeficientes variando de $0,47^{5}$ a $0,71^{20}$ para proteínas totais. Por exemplo, nos estudos de Willett et al. $(\mathrm{r}=0,47)^{3}$ e Sichieri et al. $(r=0,53$ entre auxiliares de serviços gerais e $r=0,47$ entre professores $)^{5}$, os coeficientes para proteínas totais foram próximos.

Para o colesterol, em todos os estudos os coeficientes variaram de uma correlação moderada $(r=0,61)^{3}$ a forte $(r=0,79)^{19}$, sugerindo boa validade para este nutriente.

Já para os carboidratos, houve uma discrepância importante entre as correlações. A maior $(r=0,76)$ foi encontrada por Ocké et al. ${ }^{20}$, em uma amostra de ambos os sexos, cujo objetivo era identificar fatores de risco para o câncer (European Prospective Investigation into Cancer and NutritionEPIC). Já a menor $(r=0,22)$ foi descrita por Sichieri et al..$^{5}$ em um grupo de funcionários de uma universidade brasileira.

Os coeficientes para as fibras foram bons, sendo melhores aqueles encontrados por Ocké et al. (0,61 para homens e 0,74 para mulheres $)^{20}$, e o menor por Willett et al. (0,58 para mulheres) ${ }^{3}$.

Para a vitamina A, os coeficientes foram em geral baixos, mesmo quando o método referência era um ano de registros alimentares semanais, metodologia capaz de abranger a variação sazonal e, conseqüentemente, de quantificar esse nutriente. Uma possível explicação é que esse nutriente concentra-se em grandes quantidades, mas em poucos alimentos, ocasionando uma baixa cor- relação entre um instrumento que abrange um ano de observação com os demais instrumentos, utilizados pontualmente ${ }^{6}$.

A vitamina $C$ também apresentou coeficientes de correlação bastante diferentes, variando de 0,27 em auxiliares de serviços gerais $^{5}$ a 0,71 em mulheres ${ }^{20}$.

Para os lipídeos, os coeficientes de correlação ficaram em torno de 0,60. Foi encontrada uma variação importante entre auxiliares de serviços gerais e professores no estudo de Sichieri et al. (0,34 a 0,70, respectivamente), diferença atribuída às distinções econômicas entre os grupos ${ }^{5}$.

A informação sobre o consumo do álcool apresentou uma forte correlação usando-se o QSFA e o R24 com valores de 0,85 para homens e 0,87 para mulheres ${ }^{20}$. Considerando esta adequação, reforça-se a hipótese de que o QSFA pode ser indicado para estimar sua ingestão real ${ }^{6}$.

Foi percebida durante esta revisão a inexistência de uma padronização de metodologias em estudos comparativos de métodos dietéticos. As variações vão desde a escolha do método referência até o número de repetições das mensurações e os grupos populacionais incluídos nas amostras. Estas últimas são geralmente de tamanho limitado e grupos específicos, apresentando resultados variados, limitando as inferências. Portanto, existe ainda uma grande necessidade de estudos de validação relativa em estudos de base populacionais, tanto para comparações regionais como internacionais ${ }^{3}$.

Nos estudos de validação, uma limitação bastante descrita tem sido o uso de múltiplos registros para um mesmo indivíduo. Esta tarefa é cansativa para o entrevistado, sendo mais bem aceita por voluntários, podendo assim gerar o viés de voluntariado, que por sua vez pode contribuir para a obtenção de correlações mais fortes do que na população geral, já que os voluntários podem ter motivação diferenciada para responder a múltiplos questionários. Uma alternativa para contornar tal situação tem sido a proposta do uso conjunto de estudos de validação relativa e de calibração, considerando 
que o último pode demandar apenas uma medida por indivíduo. Essa metodologia será descrita à frente.

Além dos métodos dietéticos, o uso de marcadores bioquímicos como referência tem sido proposto para validar a ingestão alimentar. Como vantagem, destaca-se que os erros de medida dos marcadores bioquímicos não estão correlacionados aos erros dos instrumentos dietéticos, como o viés de memória, por exemplo, que é uma tônica nos métodos dietéticos. Entretanto, esses marcadores possuem limitações relativas às diferenças individuais na absorção e metabolismo de nutrientes; variação intra-indivíduo; limitações das técnicas laboratoriais; custo elevado e inexistência de marcadores específicos para vários nutrientes, como carboidratos e lipídeos. Restringem-se assim os estudos utilizando-se marcadores a aspectos técnicos relativos à viabilidade e disponibilidade laboratorial ${ }^{4}$. Dessa forma, marcadores bioquímicos podem auxiliar na validação de métodos dietéticos, mas nunca devem ser a única referência.

\section{Estudos de Calibração}

A calibração é definida como o redimensionamento das medidas, isto é, a correção da ingestão obtida por um método pela comparação com o método referência, utilizando-se modelos estatísticos de regressão linear. Trata-se, portanto, de uma tentativa de ajustamento dos erros de medida provenientes do método e do grupo populacional utilizado $^{21,22}$.

A calibração tem sido usada com os seguintes objetivos: a) estimar a ingestão real através de técnicas de regressão linear $\left.{ }^{10} ; b\right)$ estimar a correlação entre a ingestão obtida a partir do QSFA e a ingestão real. Esta avaliação torna-se importante, principalmente se houver alterações do instrumento ou do grupo populacional. Se as correlações não forem satisfatórias, modificações deverão ser realizadas a fim de melhorar a adequação do instrumento; c) acessar os erros sistemáticos que podem resultar do uso do instrumento; d) e calibrar medidas de associação provenientes de exposições mensuradas pelo QSFA na presença de erros ${ }^{21}$.

Na calibração, assume-se a premissa de independência entre os erros aleatórios do questionário teste e do referência. Assim, espera-se não produzir erros sistemáticos nas estimativas do fator de calibração devido aos erros aleatórios do método referência. Portanto, a referência não precisa alcançar valores acurados da ingestão real e pode ser baseada no consumo de alimentos de um único dia. Contudo, para atingir uma adequação mínima, um número significativo de questionários deve ser obtido, seja incluindo um grande número de indivíduos ou realizando múltiplos registros para cada participante. Para obter um menor erro padrão do fator de calibração indicam-se estudos que incluam o máximo de indivíduos com uma única observação, uma vez que a variação intra-indivíduo é geralmente maior do que aquela entre indivíduos. Entretanto, a melhor escolha quanto ao número de reaplicações dependerá dos objetivos da calibração, do grau do erro de medida e do instrumento ${ }^{21,22}$.

A vantagem da calibração baseada em uma única medida por indivíduo é que esta pode ser conduzida mais facilmente em amostras populacionais, reduzindo problemas de logística e custos.

Ainda referindo-se ao Projeto Bambuí, o estudo de calibração só foi viável por contar apenas com uma única medida por indivíduo. Quanto aos resultados, na Tabela 3 estão descritas as médias corrigidas do QSFA e aquelas não corrigidas. De maneira geral, o QSFA, quando calibrado, forneceu valores menores, sendo estatisticamente significantes para o consumo de lipídeos; fibras; zinco; vitaminas C, E e A; ácidos graxos saturado, monoinsaturado e poliinsaturado; e colesterol.

Dessa forma, a definição de um bom modelo de QSFA para a mensuração local da ingestão alimentar e de nutrientes requer a confecção de estudos de validação relativa ou de calibração para que, assim, as médias de nutrientes encontradas aproximem-se do real. Essas metodologias poderão fornecer 
Tabela 3 - Comparação de medias entre o questionário semiquantitativo de freqüência alimentar associado a fotos (QSFA) corrigido e não corrigido, Bambuí - MG, Brasil, 1996-97.

Table 3 - Comparison of means between corrected and uncorrected SFFQ (semiquantitative food frequency questionnaire with photos), Bambuí-MG, Brazil, 1996-97.

\begin{tabular}{llll}
\hline Nutrientes & $\begin{array}{l}\mathrm{QSFA}_{\text {não corrigido }}(\mathrm{n}=98) \\
\text { Média } \pm \text { desvio padrão }\end{array}$ & Valor $\mathrm{p}^{(1)}$ & $\begin{array}{l}\mathrm{QSFA}_{\text {corrigido }}(\mathrm{n}=98) \\
\text { Média } \pm \text { desvio padrão }^{(1)}\end{array}$ \\
\hline Calorias & $3187,1 \pm 1394,2$ & 0,19 & $2975,4 \pm 738,6$ \\
Proteínas & $121,7 \pm 61,7$ & 0,08 & $110,1 \pm 24,0$ \\
Lipídeos & $107,4 \pm 68,6$ & 0,04 & $92,2 \pm 25,6$ \\
Carboidratos & $431,2 \pm 167,4$ & 0,14 & $404,1 \pm 65,4$ \\
Fibras & $9,3 \pm 6,4$ & 0,01 & $7,6 \pm 1,8$ \\
Cálcio & $1172,4 \pm 712,1$ & 0,08 & $1039,0 \pm 256,2$ \\
Fósforo & $1759,4 \pm 837,2$ & 0,12 & $1615,0 \pm 383,1$ \\
Ferro & $22,8 \pm 11,4$ & 0,07 & $20,6 \pm 4,1$ \\
Zinco & $13,2 \pm 8,2$ & 0,03 & $11,3 \pm 2,3$ \\
Vitamina C & $378,2 \pm 270,4$ & 0,02 & $306,7 \pm 107,0$ \\
Vitamina B & $3,3 \pm 1,5$ & 0,06 & $3,0 \pm 0,5$ \\
Vitamina E & $11,1 \pm 5,5$ & 0,04 & $9,9 \pm 1,8$ \\
Vitamina A & $1210,5 \pm 880,0$ & 0,02 & $985,0 \pm 258,3$ \\
Ácido Graxo saturado & $36,4 \pm 25,6$ & 0,01 & $29,7 \pm 6,3$ \\
Ácido graxo poliinsaturado & $6,3 \pm 3,5$ & 0,02 & $5,4 \pm 1,3$ \\
Ácido graxo monoinsaturado & $15,8 \pm 11,1$ & 0,01 & $12,8 \pm 3,1$ \\
Colesterol & $369,3 \pm 265,6$ & 0,01 & $301,6 \pm 83,1$ \\
\hline
\end{tabular}

(1) Teste T para comparação de medias pareadas entre QSFA e QSFA ${ }_{\text {calibrated }}$

medidas de exposição mais acuradas e gerar associações mais claras entre dieta e eventos, contribuindo para o entendimento etiológico de eventos.

\section{Agradecimentos}

Programa de Saúde Pública da Universi- dade Federal de Minas Gerais, Centro de Pesquisas Rene Rachou da Fundação Oswaldo Cruz, Comissão de Aperfeiçoamento de Pessoal Docente de Nível Superior, Fundo de Amparo à Pesquisa do Estado de Minas Gerais e Conselho Nacional de Desenvolvimento Científico e Tecnológico pelo apoio financeiro e logístico.

\section{Referências}

1. Gibson RS. Principles of nutritional assessment. New York: Oxford University Press; 1990.

2. Mahan L, Kathleen ESS. Krause: Alimentos, Nutrição \& Dietoterapia. 10a ed. São Paulo: Roca, 2002.

3. Willett WC, Sampson L, Stampfer MJ et al. Reproducibility and validity of a semiquantitative food frequency questionnaire. Am J Epidemiol 1985; 122: 51-65.

4. Willett WC. Nutritional Epidemiology. New York: Ed. Oxford University Press; 1998.
5. Sichieri R. Epidemiologia da obesidade. Rio de Janeiro: EdUERJ; 1998.

6. Pereira RA, Koifman S. Uso do Questionário de Freqüência na Avaliação do Consumo Alimentar Pregresso. Rev Saúde Pública 1999; 33(6): 610-21.

7. Reniscow K, Odom E, Wang T, Dudley WN et al. Validation of Three Food Frequency Questionnaires and 24-Hour Recalls with Serum Carotenoid Levels in a Sample of African-American Adults. Am J Epidemiol 2000; 152: 1072-9. 
8. Spiegelman D, McDermott A, Rosner B. Regression calibration method for correcting measurement-error bias nutritional epidemiology. Am J Clin Nutr 1997; 65: S1179-86.

9. Lopes ACS. Projeto Bambuí: Avaliação de Instrumental Metodológico para Uso em Inquéritos Nutricionais Calibrando o Questionário Semiquantitativo de Freqüência Alimentar (QSFA) em um Inquérito Populacional [dissertação de mestrado]. Universidade Federal de Minas Gerais. 1999.

10. Beaton GH, Burema J, Ritenbaugh C. Errors in the interpretation of dietary assessments. Am J Clin Nutr 1997; 65: S1100-7.

11. Rimm EB, Giovannucci EL, Stampfer MJ et al. Reproducibility and validity of a expanded selfadministered semmiquantitative food frequency questionnaire among males health professionals. Am J Epidemiol 1992; 135: 1114-26.

12. Byers TE, Roshental RI, Marshall TF et al. Dietary history from the distant past: a methodolgical study. Nutr Cancer 1983; 5: 69-77.

13. Byers TE, Marshall E, Anthony R et al. The realibility of dietary history from the distant past. Am J Epidemiol 1987; 125: 999-1011.

14. Pietinen P, Hartman AM, Haapa E et al. Reproducibility and validity on dietary assessment instruments: i. a self-administers food use questionnaire with a portion size picture booket. Am J Epidemiol 1988; 128; 655-66.

15. Engle A, Lynn LL, Koury K, Boyar AP. Reproducibility and comparability of a computadorized, selfadministred food frequency questionnaire. Nutr Cancer 1990; 13: 281-92. In: Willett WC. Nutritional Epidemiology. New York: Ed. Oxford University Press; 1998.
16. Riley MD, Blizzard L. Comparative Validity of a Food Frequency Questionnaire for Adults with IDDM. Diabetes Care. Am J Epidemiol 1995; 18: 1249-54. In: Willett WC. Nutritional Epidemiology. New York: Ed. Oxford University Press; 1998.

17. Hansson LM, Galanti MR Bergström R. Factors Affecting Reproducibility of Dietary Reports Using Food Frequency Questionnaires. European J Clin Nutr 2000; 54: 658-64.

18. Kaaks R, Riboli E. Validation and calibration of dietary intake measurements in the EPIC project: methodological considerations. Int J Epidemiol 1997; 26: S15-24.

19. Browe JH, Gofstein MR, Morlley DM, McCathy MC. Diet and Heart Disease study in the Cardiovascular Health Center. J Am Diet Assoc 1966; 48: 95-100.

20. Ocké MC, Bueno-de-Mesquita BH, Margreet PA et al. The Dutch EPIC food frequency questionnaire relative validity and reproducibility for nutrients. Int $J$ Epidemiol 1997; 26: S49-58.

21. Stram DO, Hankin JH, Wilkens SR, Pike MC et al. Calibration of Dietary Questionnaire for a Multiethnic Cohort in Hawaii and Los Angeles. Am J Epidemiol 2000; 151(4): 358-70.

22. Slimani N, Ferrari P, Ocké M, Welch A et al. Santardization of the 24-hour Recall Calibration Method Used in the European Prospective Investigation into Cancer and Nutrition (EPIC): General Concepts and Preliminary Results. European $J$ Clin Nutr 2000; 54: 900-17.

Recebido em: 07/03/2002 Versão final reapresentada em: 13/05/2003 Aprovação em: 26/05/2003 